\title{
A PROPOS DU PHLEBOTOMUS CHINENSIS
}

\author{
Par Virgil NITZULESCU
}

Notre collègue T. Simic vient de publier dans ces Annales (1), une note consacrée à l'étude complémentaire des phlébotomes de Skoplje - et surtout du P. chinensis que nous avions signalé en Yougoslavie en 1929. Nous n'avions pu étudier, lors de notre note de 1929, que trois mâles et quelques femelles de $P$. chinensis de Skoplje; au contraire, Simic a pu disposer d'un lot de 170 mâles et 217 femelles de cette espèce. Nous avons été heureux de voir que toutes nos observations sont confirmées par notre savant collègue et ami. Il y a toutefois certains caractères, dans la note de T. Simic, qui auraient besoin, d'après nous, d'être revus.

D'après Simic, le tubercule subapical inférieur de l'organe intromittent varie de grandeur et de forme, suivant que les filaments génitaux sortent ou ne sortent pas par cet organe. Si cette observation était exacte, elle enlèverait, du même coup, toute l'importance taxonomique que nous croyons devoir rattacher à la forme et à la position de ce tubercule. A notre avis, la forme et grandeur du dit tubercule n'ont aucune relation avec les filaments génitaux. L'organe intromittent, qui est appelé aussi par quelques auteurs " valve copulatrice ", joue en effet le rôle d'une valve ou d'un fourreau, dans lequel glissent les filaments génitaux, dans leurs mouvements de sortie et de rentrée. Il n'y a aucune adhérence entre les filaments génitaux et les valves copulatrices et, par conséquent, aucun rapport entre les filaments génitaux et le tubercule qui garnit la face externe et inférieure du fourreau. La forme du tubercule subapical de l'organe intromittent varie en effet avec la position de l'organe : il ne s'agit pas alors d'une variation réelle, mais d'une apparence différente, en fonction de l'angle sous lequel on l'examine (vue de profil, de trois-quarts, ou de face).

La touffe de poils du premier segment de la gonapophyse supérieure est, en effet, comme le remarque Simic, bien développée dans les exemplaires de Skoplje. Nous l'avons d'ailleurs signalée et dessinée dans notre note, mais nous étions alors de l'avis qu'elle est moins développée que dans les exemplaires de Bucarest. Cette opi-

(1) IX, 1931, p. 104-110.

Annales de Parastrologie, T. IX, $n^{\circ} 3 .-1^{\text {er }}$ mai 1931, p. 261-265. 
nion se trouve confirmée par l'examen d'un lot important de $P$. chinensis de Skoplje, que Simic vient d'envoyer au laboratoire de Parasitologie de la Faculté de Médecine de Paris. Il y a une différence remarquable entre la touffe de poils du $P$. chinensis de Bucarest, si développée qu'elle fait comme une tache noire sur le jaune clair de la gonapophyse et la touffe moins développée du $P$. chinensis de Skoplje. Par contre, la touffe de poils correspondante dans la variété authentique de Chine est encore bien moins développée (fig. 8).

Un autre point sur lequel nous ne nous rattachons pas à l'avis de Simic concerne les antennes. D'après cet auteur, on ne peut tirer des antennes aucun caractère utile pour la différenciation des phlébotomes de Bucarest, de Skoplje et de Chine. Or, nous avons eu déjà l'occasion d'attirer l'attention à deux reprises sur l'étroite corrélation qui existe entre la forme et la disposition des épines géniculées des antennes et la morphologie de l'organe intromittent. Premièrement, en décrivant les deux types du P. brumpti (Nitzulescu, 1930, a) qui sont représentés dans la collection du Laboratoire de Parasitologie de la Faculté de Médecine de Paris ; deuxièmement, en décrivant la nouvelle espèce $P$. langeroni et sa variété longicuspis (Nitzulescu, $1930, b$ ) de Douar-Shott. Quant au $P$. chinensis, nous avons déjà mentionné, dans notre note consacrée à la description de la forme de Chine, que cette forme présente la formule $\frac{2}{\mathrm{III}-\mathrm{XV}}$, tandis que la forme de Bucarest présente la formule $\frac{2}{\text { III-VIII }}, \frac{1}{\text { IX-XV }}$. La formule antennaire de la forme de Skoplje est pareille à celle de la forme de Chine.

Les femelles des phlébotomes de Skoplje présentent, elles aussi, un caractère plus ou moins nettement visible, résultant de la forme même des spermathèques, toujours plus allongées dans la forme de Skoplje que dans celles de Bucarest ou de Chine.

Séparation du P. chinensis en variétés distinctes. - Après avoir étudié, en 1929, les trois phlébotomes mâles de Skoplje et le grand lot que Mme le $\mathrm{D}^{r} \mathrm{G}$. Nitzulescu venait de découvrir à Bucarest, nous avions émis des doutes concernant la variabilité des deux formes, celle de Bucarest et celle de Skoplje. Les variations ne nous paraissaient pas tenir à la simple plasticité de l'espèce, mais nous avions l'impression de nous trouver en face de deux types bien caractérisés. Toutefois, le peu de matériel que nous avions reçu de Skoplje ne nous permettait pas d'être trop catégorique à cet égard. L'étude récente de Simic et le lot important qu'il a envoyé à Paris et que nous avons eu la possibilité d'étudier par nous-même, a 
confirmé pleinement ce point de vue, car tous les exemplaires nouveaux présentent exactement les mêmes caractères que ceux que nous avions eu à étudier en 1929. D'autre part, l'étude des exemplaires authentiques de Chine nous a démontré l'existence d'une troisième forme à laquelle se rattachent tous les exemplaires examinés et que nous avons déjà décrite dans un numéro précédent de ces Annales (1).

Pour le reste de la vaste région géographique sur. laquelle le $P$. chinensis est répandu, nous avions exprimé, dès 1929, le désir de savoir -si, là aussi, les variations du $P$. chinensis, déjà signalées par Adler et Theodor, ne se groupent pas sous la forme de types. Nous venons de trouver ce souhait réalisé dans un récent article d'Adler, Theodor et Lourie. Ces auteurs trouvent, en effet, qu'il y a en Palestine, en Syrie et en. Perse des races locales comparables à celles d'Europe, c'est-à-dire que la forme de Perse et de Syrie est identique à la forme de Bucarest et celle de Yougoslavie est presque la même que cèlle de Palestine.

Ainsi donc, si chaque organe du $P$. chinensis peut varier entre certaines limites, ces variations sont en corrélation mutuelle. Il ne s'agit plus alors de variations d'organes, mais de variétés vraies de la même espèce sinon de plusieurs espèces distinctes.

L'expression de " races locales " d'Adler, Theodor et Lourie ne nous parait pas appropriée. Les différences sont plus nettes qu'entre deux races. Il existe, certainement, plus de différence entre le $P$. chinensis de Bucarest et le $P$. chinensis de Chine, qu'entre $P$. major et $P$. wenyoni ou entre $P$. perniciosus et sa variété tobbi. D'ailleurs il nous parait difficile de parler de " races locales " quand il s'agit de formes identiques en Perse et à Bucarest d'un côté, en Palestine et en Yougoslavie de l'autre côté.

Il est grand temps, en conséquence, de procéder au démembrement, en variétés distinetes, de cette espèce si répandue, démembrement qui pourra rendre de grands services à ceux qui voudront étudier de plus près la biologie du $P$. chinensis.

L'espèce-type doit rester celle de Chine que nous avons décrite en 1929. L'organe intromittent y est plus court que les appendices intermédiaires et le tubercule inférieur de l'extrémité distale est assez éloigné de l'apex et aplati. La longueur des filaments génitaux varie entre 5,5 et 7 fois la longueur de la pompe génitale. La touffe de poils est très peu marquée. Les épines géniculées des antennes du mâle sont disposées d'après la formule $\frac{2}{\text { III-XV}}$. La spermathèque

(1) VIII, 1930, p. 362-375. 
des femelles, d'après Sinton, présente des étranglements bien visibles quoique non profonds.

La forme de Bucarest, de Perse et de Syrie avait été séparée par Parrot en 1928. Elle avait déjà été nommée $P$. major var. longiductus, à cause de la longueur excessive des filaments génitaux (dix fois la longueur de la pompe). Le pénis y est plus court que les appendices intermédiaires. Le tubercule subapical est plus rapproché de l'apex et plus développé. La touffe de poils est très développée et noire (fig. 9 et 10). Les épines géniculées des antennes possèdent la formule

$$
\frac{2}{\text { III-VIII }}, \quad \frac{1}{\text { IX-XV }} \text {. }
$$

La forme de Bucarest, de Perse et de Syrie doit prendre le nom de $P$. chinensis var. longiductus (Parrot, 1928). Au sein de cette variété, la forme de Syrie, avec une pigmentation plus accentuée, pourra être considérée comme une " race locale ".

La forme de Yougoslavie diffère des deux formes précédentes par la configuration du pénis qui est très allongé et présente un tubercule subapical vrai, c'est-à-dire situé tout à fait à l'extrémité distale. La formule antennaire et les filaments génitaux sont semblables à ceux de la forme de Chine et nettement différents de ceux de la

\section{Explication des Planches}

Planche V

Fig. 1. - Organe intromittent du Phlebotomus chinensis de Chine.

Fig. 2. - Organe intromittent du Phlebotomus chinensis, var. longiductus de Bucarest.

Fig. 3. - Organe intromittent du Phlebotomus chinensis, var. simici de Yougoslavie.

FıG. 4. - Spermathèque du Phlebotomus chinensis, var. simici de Yougoslavie.

Planche VI

Fıg. 5 et 6. - Spermathèques du Phlebotomus chinensis, var. longiductus de Bucarest.

Fig. 7. - Appareil génital mâle du Phlebotomus chinensis, var, simici de Yougoslavie.

Fıg. 8. - Appareil génital mâle du Phlebotomus chinensis, de Chine.

PLANChe VII

Fı́. 9. - Appareil génital mâle du Phlebotomus chinensis var. longiduclus de Bucarest.

Fig. 10. - Touffe de poils du premier segment de la gonapophyse supérieure chez le Phlebotomus chinensis, var. longiductus de Bucarest. 


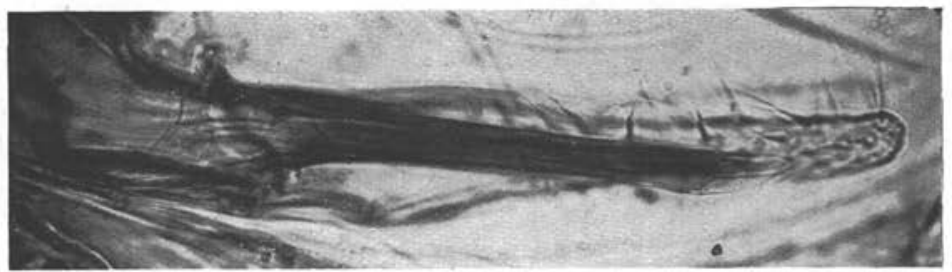

Fig. 1.

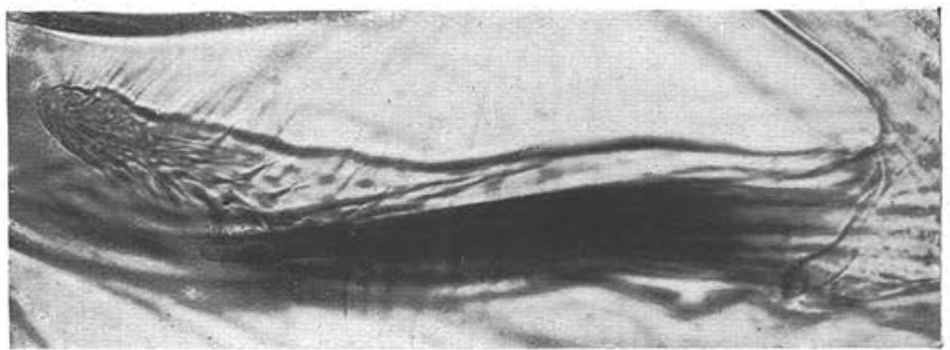

Fig. 2.

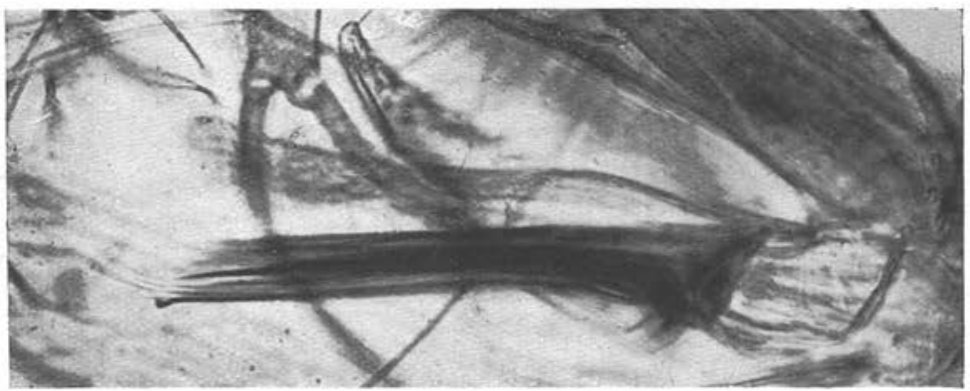

F1G. 3.

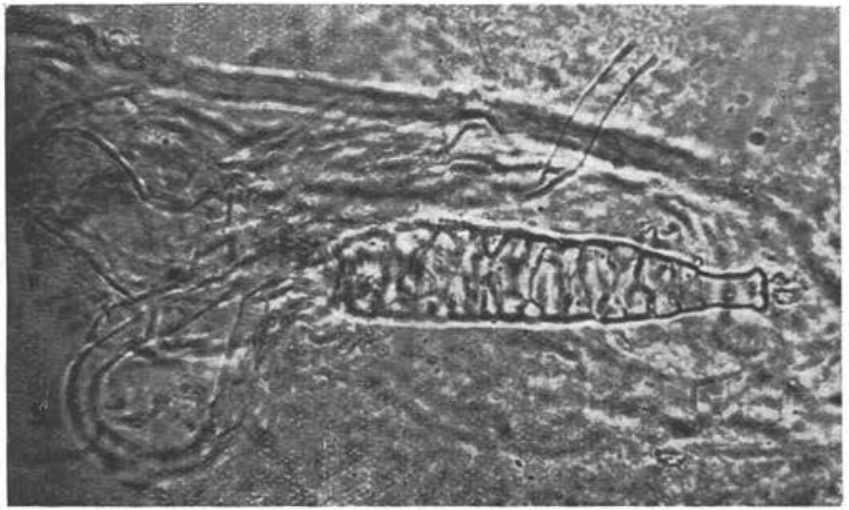

Fig. 4.

Masson et Cie, Editeurs 

ANNALES DE PARASITOLOGIE

T. IX, No 3, mai 1931.
Planche VI

(Némoire Nitzulescu).

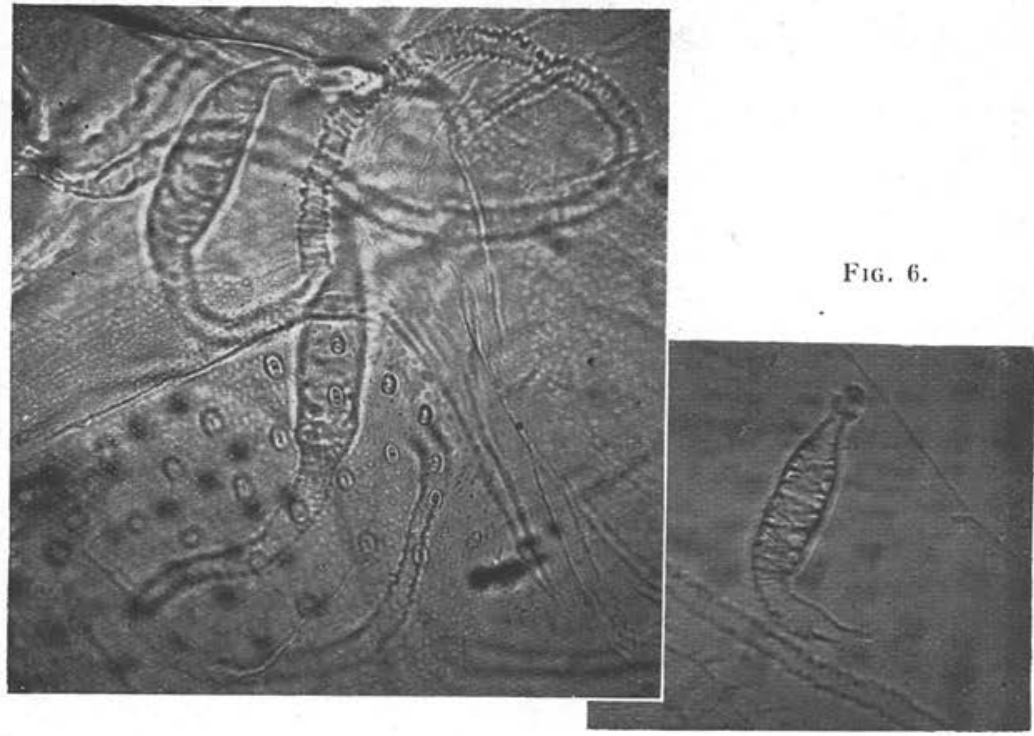

Fig. 5.

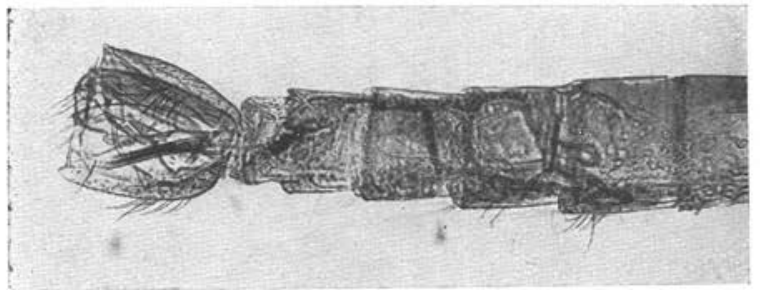

Fig. 7.

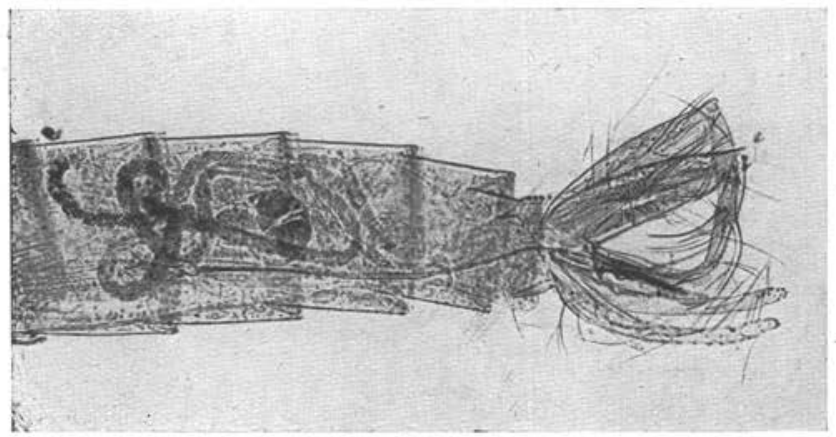

FIG. 8.

Masson et Cie, Editeurs 

ANNALES DE PARASITOLOGIE

T. IX, No 3, mai 1931.
Planche VII

(Mémoire Nitzulescu).

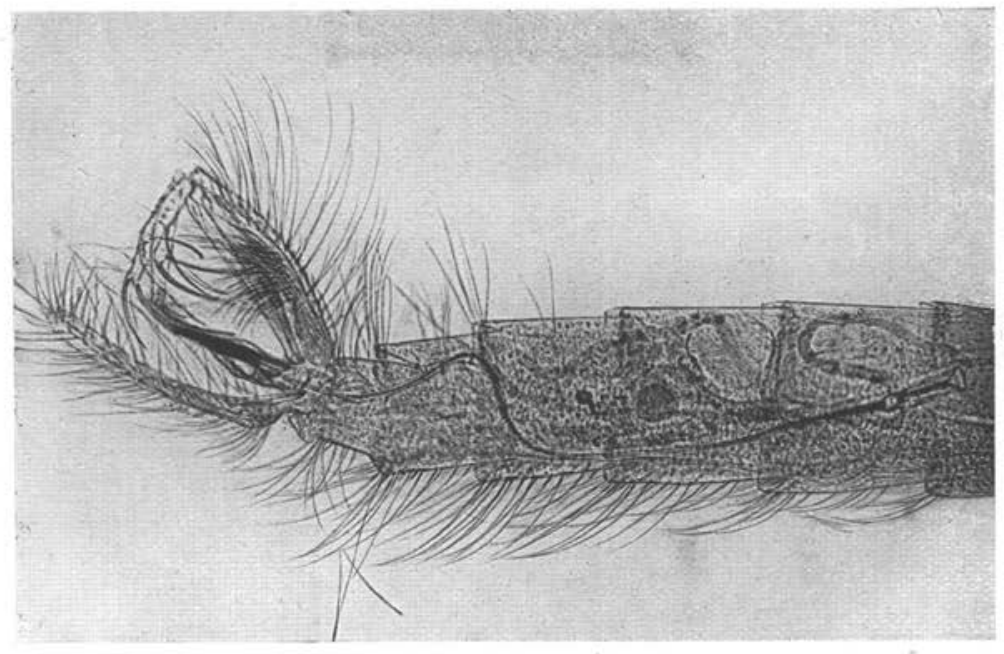

Fig. 9.

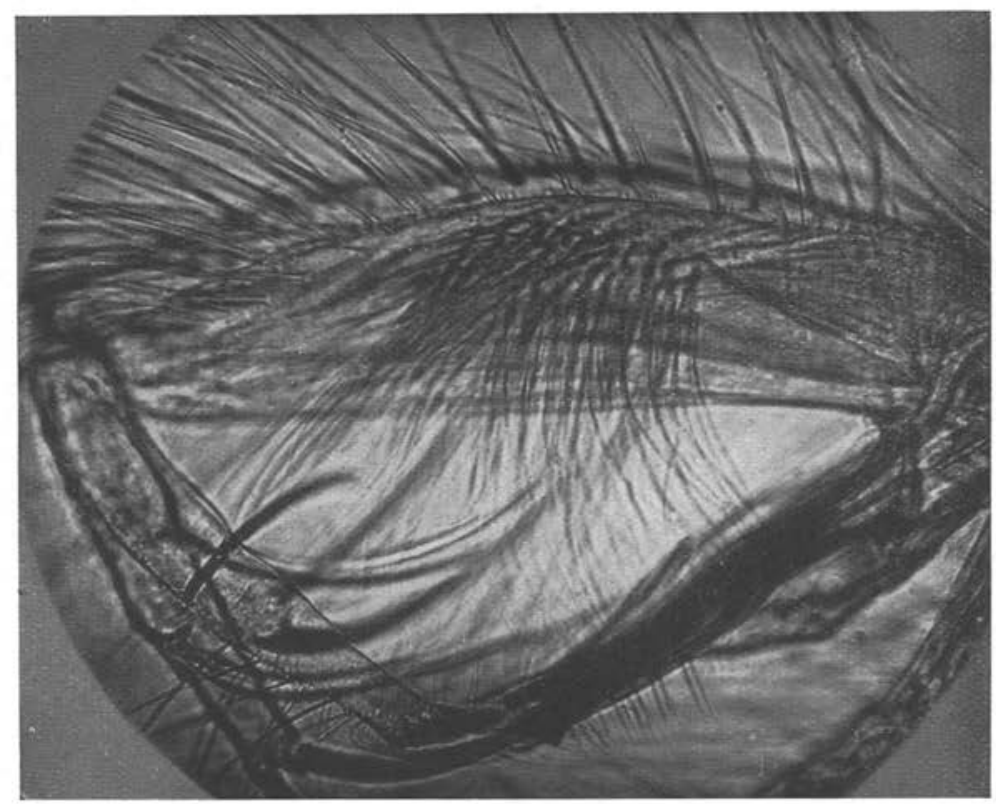

FIG. 10 .

Masson et Cie, Editeurs 

forme de Bucarest. Les dents pharyngiennes sont très noires (1). La touffe de poils, moins développée que dans la forme de Bucarest, est plus développée que dans la forme de Chine (fig. 7). C'est à cette variété, que nous avons pu étudier, gràce au matériel envoyé par mon collègue et ami, le $\mathrm{D}^{r}$ Simic, de Skoplje, que nous proposons de donner le nom de $P$. chinensis var. simici.

Cette variété se trouve en Yougoslavie, et aussi en Palestine, où il y aurait, d'après Adler, Theodor et Lourie, une race plus petite.

Nous complétons l'iconographie des variétés de $P$. chinensis que nous avons déjà décrites en détail dans des notes antérieures, par quelques microphotographies, que nous devons à la grande amabilité du $\mathrm{D}^{\mathrm{r}}$ M. Langeron, et qui reproduisent fidèlement les caractères particuliers ci-dessus indiqués.

\section{Bibliographie}

Ader et Theonor. - The distribution of sandflies and leishmaniasis in Palestine, Syria and Mesopotamia. Annals of tropical medecine and parasitology, XXIII, 1929, p. 269-306.

Adeer, Theodor et Lourie. - On sandflies from Persia and Palestine. Bull, ent. res., XXI, 1930, p. 529-539.

Nitzulescu (V.). - Contribution à l'étude des phlébotomes de Roumanie. Ann. de Parasit., VII, 1929, p. 320-437.

- Sur quelques phlébotomes de Yougoslavie. Ann. de Parasit., VII, 1929, p. 49t-505.

- Sur le Phlebotomus chinensis. Ann. de Parasit., VIII, 1930, p. 363-374.

- Sur le Phlebotomus troglodyles et le Phlebotomus brumpti. Ann. de Parasit., VIII, 1930 , p. $386 \cdot 393$.

Srmic (T.). - Etude complémentaire des phlébotomes de Skoplje. Ann. de Parasit., IX, 1931, p. 104-110.

Laboraloire de Parasilologie de la Faculté de Médecine de Paris

(1) Nous corrigeons ici un lapsus calami qui nous avait échappé pendant la correction de notre note sur le P. chinensis. Nous disions que le pharynx de la forme de Chine est moins coloré que celui des formes européennes et surtout que celui de la forme de Bucarest. C'est de la forme de Skoplje que nous avons voulu parler, car c'est elle qui possède les dents pharyngiennes les plus noires. 